Physics Header

\title{
Phase-separation of miscible liquids in a centrifuge
}

\author{
Yoav Tsori ${ }^{\mathrm{a}}$, Ludwik Leibler ${ }^{\mathrm{b}}$ \\ ${ }^{a}$ Department of Chemical Engineering, Ben-Gurion University of the Negev, \\ P.O. Box 653, 84105 Beer-Sheva, Israel \\ ${ }^{\mathrm{b}}$ Laboratoire Matière Molle $\mathcal{E}$ Chimie (UMR 7167), ESPCI, \\ 10, rue Vauquelin, 75231 Paris CEDEX 05, France \\ Received *****; accepted after revision +++++
}

\begin{abstract}
We show that a liquid mixture in the thermodynamically stable homogeneous phase can undergo a phaseseparation transition when rotated at sufficiently high frequency $\omega$. This phase-transition is different from the usual case where two liquids are immiscible or where the slow sedimentation process of one component (e.g. a polymer) is accelerated due to centrifugation. For a binary mixture, the main coupling is due to a term $\propto \Delta \rho(\omega r)^{2}$, where $\Delta \rho$ is the difference between the two liquid densities and $r$ the distance from the rotation axis. Below the critical temperature there is a critical rotation frequency $\omega_{c}$, below which smooth density gradients occur. When $\omega>\omega_{c}$, we find a sharp interface between the low density liquid close to the center of the centrifuge and a high density liquid far from the center. These findings may be relevant to various separation processes and to the control of chemical reactions, in particular their kinetics.
\end{abstract}

\section{Séparation de phases des liquides miscibles dans une centrifugeuse}

Résumé

Nous prouvons qu'un mélange de liquides dans une phase homogène thermodynamiquement stable peut subir une transition de phase une fois centrifugé á une fréquence $\omega$ suffisamment élevée. Cette transition de phase est différente du cas habituel où deux liquides sont non-miscibles ou du cas où un processus lent de sédimentation d'un composant (par exemple un polymère) est accéléré par une centrifugation. Pour un mélange binaire, le couplage principal est dû à un terme $\propto \Delta \rho(\omega r)^{2}$, où $\Delta \rho$ est la différence des densités entre les deux liquides et $r$ est la distance de l'axe de rotation. Au-dessous de la température critique, il y a une rotation critique de fréquence $\omega_{c}$; au-dessous d' $\omega_{c}$, de faibles gradients de densité prennent naissance. Quand $\omega>\omega_{c}$, nous trouvons une interface mince entre le liquide de faible densité près du centre de la centrifugeuse et un liquide à haute densité loin du centre. Ces résultats peuvent être appropriés à divers processus de séparation et au contrôle de réactions chimiques, en particulier de leur cinétique.

Key words: Centrifuge; Phase-transition ; Critical frequency

Mots-clés : Centrifugeuse; transition des phases; fréquence critique

Email addresses: tsori@bgu.ac.il (Yoav Tsori), ludwik.leibler@espci.fr (Ludwik Leibler). 


\section{Introduction}

The phase of a liquid mixture typically depends on the ambient temperature, pressure, and composition, but may also be influenced by external fields such as magnetic and electric fields $[1,2,3,4,5]$, surface fields [6,7], or gravity $[8,9,10,11,12,13]$. The gravitational field couples to the density of the different components, and thus tends to separate them $[14,15,16]$. Since this coupling is rather weak, gravity is mainly associated with the slow sedimentation process of an already immiscible mixture. A common way to accelerate this sedimentation is by the use of centrifugation, where the high achievable rotation frequencies permit an effective acceleration highly superior to the simple gravitation case $[17,18]$. Since centrifugation is very effective as an aid to separation of substances which are not thermodynamically mixed, it is rarely used on thermodynamically homogeneous mixtures. Indeed, as we show below, when a homogeneous mixture is rotating around some symmetry axis, only small composition gradients appear in the radial direction with respect to this axis [18]. However, and this is a surprising result of our analysis, if the mixture is rotating rapidly enough, above a threshold value, the mixture composition exhibits a sharp jump from one composition to another; this sharp change is the signature of a phase-separation. This phase separation should be accessible for many mixtures using modern ultracentrifugation techniques. A simple theory is developed, analyzed and discussed below.

\section{Theory and Results}

The mixture under consideration here is binary and symmetrical, consisting of two polymers A and B, each made up of $N$ identical monomers of volume $v_{0}$. The case of simple liquids is obtained when $N=1$. The local volume fraction of the A molecules is $\phi(0<\phi<1)$, and the dimensionless Flory parameter $\chi \sim 1 / T$ characterizes the repulsion between the species. The average mixture composition is denoted $\phi_{0}$ and the mass density is $\rho_{0}$. We further assume a linear constitutive relation between composition and density, namely

$$
\rho(\phi)=\rho_{0}+\Delta \rho\left(\phi-\phi_{0}\right)
$$

where $\Delta \rho \equiv \rho_{A}-\rho_{B}$, and $\rho_{A}$ and $\rho_{B}$ are the densities of the A and B components, respectively. 1 The starting point of our formulation for a mixture rotating in an angular frequency $\omega$ around some axes is the following free-energy density

$$
f=\frac{k_{B} T}{v_{0}} f_{m}(\phi)-\frac{1}{2} \rho(\phi)(\omega r)^{2}
$$

In the above $f_{m}$ is the Flory-Huggins free energy of mixing,

$$
f_{m}(\phi)=\frac{1}{N} \phi \ln (\phi)+\frac{1}{N}(1-\phi) \ln (1-\phi)+\chi \phi(1-\phi)+\frac{1}{2} \xi^{2}(\nabla \phi)^{2}
$$

where $k_{B}$ is the Boltzmann constant and $T$ is the temperature. The last term, proportional to $(\nabla \phi)^{2}$, is due to an energy penalty for having composition gradients. It gives rise to a characteristic length scale of phaseseparated domains scaling as $\sim\left(T_{c}-T\right)^{-1 / 2}$, diverging at the critical point [19]. In the discussion below, we are not restricted to mixtures close to the critical point, and therefore this term is subsequently neglected. The phasediagram derived from this mixing energy has a critical point at $\left(\phi_{c}, \chi_{c}\right)=(1 / 2,2 / N)$. The transition (binodal) value of $\chi$ at a given composition $\phi$ is given by

$$
N \chi_{t}=\frac{1}{2 \phi-1} \ln \left(\frac{\phi}{1-\phi}\right)
$$

We may write to a good approximation $T=B / \chi$, where $B$ is a constant with units of $\mathrm{K}$, and in this case the critical temperature is $T_{c}=B N / 2$ and the transition temperature is $T_{t}=B / \chi_{t}$. We are interested in mixtures in the homogeneous phase, at temperatures $T$ below $T_{c}$ but above $T_{t}$, and off-critical compositions $(\phi \neq 1 / 2)$. The second term in Eq. (2), proportional to $(\omega r)^{2}$, is due to the centrifugal forces. Here $r$ is the distance from the rotation axes, and is bounded by the smallest and largest radii: $R_{1}<r<R_{2}$.

\footnotetext{
1 Our theory does not depend on the linear relation between mass density and composition. Quadratic and higher order dependences lead to qualitatively similar conclusions.
} 


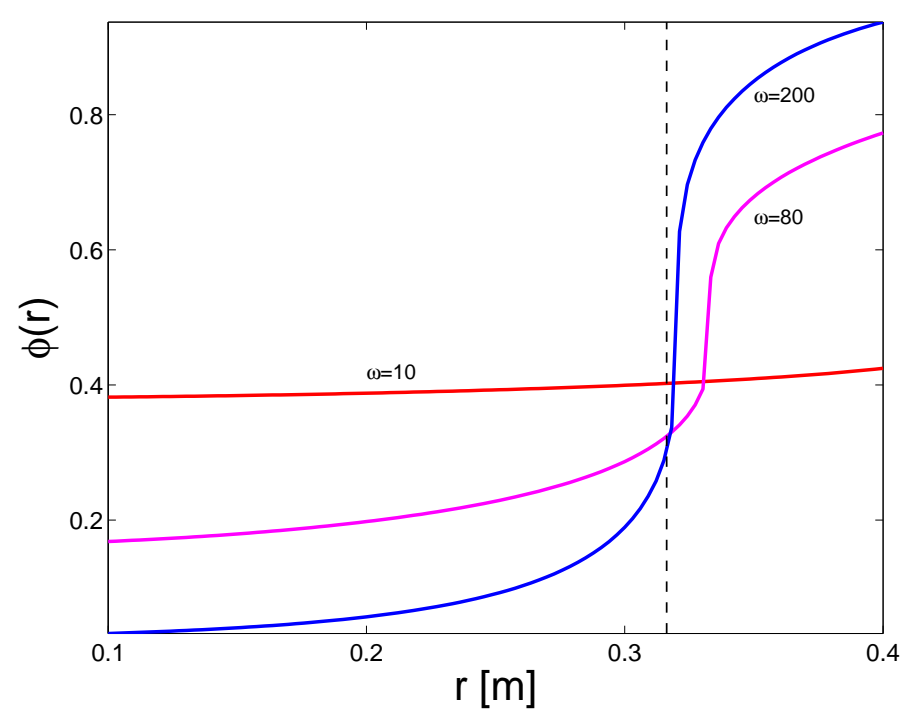

Figure 1. Composition profiles for a binary liquid mixture confined in a tube with inner radius $R_{1}=10 \mathrm{~cm}$ and outer radius $R_{2}=40$ $\mathrm{cm}$, as obtained from Eqs. (5) and (6) with $\chi_{c}<\chi<\chi_{t}\left(T_{c}>T>T_{t}\right)$. At low rotation frequencies $\omega=10 \mathrm{~s}^{-1}$, the high-density component is pulled toward the larger radius, and the resulting profile exhibits weak gradients. If the rotation frequency is large enough, $\omega=80 \mathrm{~s}^{-1}>\omega_{c}$, a sharp interface separating large and small $\phi$-values appears. As the frequency is further increased to $\omega=300 \mathrm{~s}^{-1}$, the difference between coexisting domains becomes larger, and the front moves to smaller values of $r$. The horizontal dashed line is the lowest possible value of the front location $R_{\min }$ [Eq. 7)], corresponding to maximal phase-separation. We used $N=200, v_{0}=2 \times 10^{-28} \mathrm{~m}^{3}, \rho_{0}=1000 \mathrm{Kg} / \mathrm{m}^{3}, \Delta \rho=100 \mathrm{Kg} / \mathrm{m}^{3}, \phi_{0}=0.4, T_{c}=80^{\circ} \mathrm{C}$ and the temperature is $T-T_{t}=3.5^{\circ} \mathrm{K}$.

In the presence of Earth's gravity alone, such an inertia term is absent and one has to consider buoyancy effects only very close to the consolute point. However, as we will show below and in particular for modern centrifuges rotating at high speeds, these inertia effects are important and can modify the thermodynamic aspects of separation phenomena.

We minimize the free-energy in Eq. (2) to obtain the following governing equation for the profile $\phi(\mathbf{r})$ :

$$
\frac{k_{B} T}{N v_{0}}\left[\ln \left(\frac{\phi}{1-\phi}\right)+N \chi(1-2 \phi)\right]-\frac{1}{2} \Delta \rho(\omega r)^{2}-\mu=0
$$

$\mu$ is the Lagrange multiplier (chemical potential) required to conserve the average composition of a system enclosed in a volume $V$ :

$$
\frac{1}{V} \int \phi(\mathbf{r}) \mathrm{d}^{3} r=\phi_{0}
$$

Eq. (5) gives an analytical inverted expression for the profile $r$ as a function of $\phi$.

In Fig. 1 we display the calculated profile $\phi(r)$ for several angular frequencies $\omega$. Before centrifugation, the mixture is initially homogeneous, and thus $\chi<\chi_{t}\left(T>T_{t}\right)$. The centrifugal term in Eq. (5) is spatially-dependent, and this means that when the mixture is rotated, $\phi(r)$ has smooth density changes: high-density material is pulled toward large radii whereas small density material is pulled toward small $r$. This behavior is found for $\chi<\chi_{c}$, irrespective of $\omega$. A different scenario occurs for $\chi_{c}<\chi<\chi_{t}$, which corresponds to a temperature lower than the critical temperature but higher than the binodal. At this regime, if $\omega$ is small, smooth composition variations occur. However, there exist a critical rotation frequency $\omega_{c}$, above which a phase-separation transition takes place. If $\omega>\omega_{c}, \phi(r)$ has a jump from large values at large $r$ to low values at small $r$. Note that $\phi(r)$ is not constant in each of the domains, but instead has a weak gradient, as is seen in Fig. 1. The equilibrium location of the front separating the two domains, $R$, moves to smaller $r$ values as the rotation frequency is further increased above $\omega_{c}$. As $R$ decreases, the difference between the compositions in the two domains increases. $R$ has a lower bound denoted $R_{\text {min }}$, which corresponds to the maximal possible separation between the two liquids: pure A component is found at large $r$ 's and pure B is closer to the centrifuge's axes. It follows that $R_{\min }$ obeys

$$
R_{\min }^{2}=R_{2}^{2}\left(1-\phi_{0}\right)+R_{1}^{2} \phi_{0}
$$




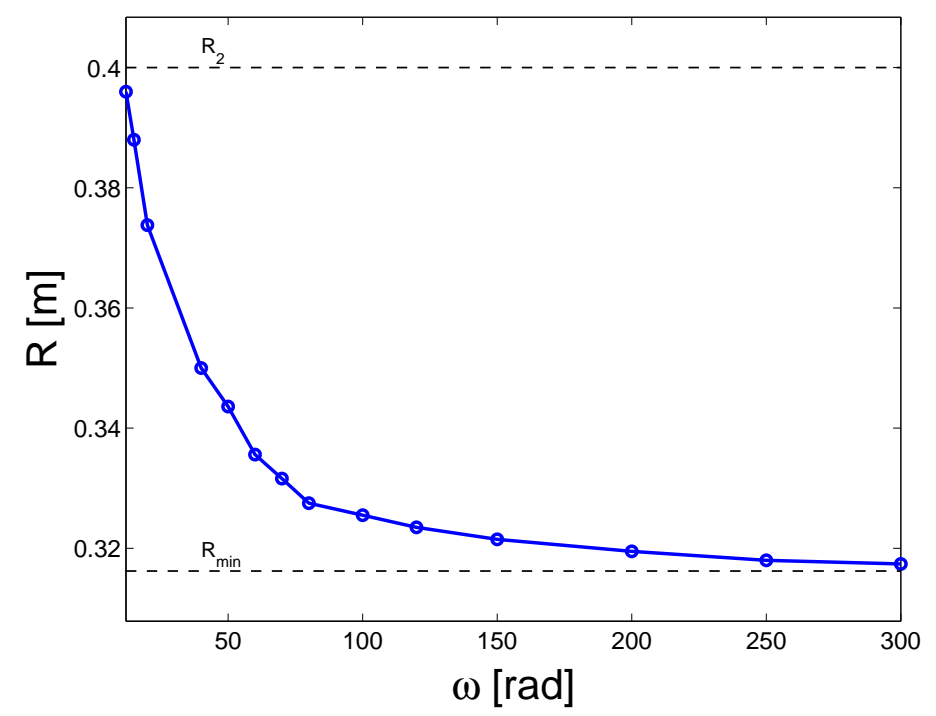

Figure 2. Equilibrium front location $R$ separating high- and low- $\phi$ domains as a function of angular frequency $\omega$, as obtained from Eqs. (5) and (6). $R$ is smaller than $R_{2}$ and decreases as $\omega$ increases above $\omega_{c} \simeq 12 \mathrm{~s}^{-1}$; it tends to $R_{\mathrm{min}}[$ Eq. (7)] for very large $\omega$. The approximate expression for $\omega_{c}$, Eq. (9), gives $\omega_{c}=15 \mathrm{~s}^{-1}$. All parameters and constants are the same as in Fig. 1.

In order to obtain an analytical expression for the critical frequency $\omega_{c}$, we continue with the following approximation. We expand Eq. (5) to linear order in $\phi-\phi_{0}$, and substitute in Eq. (6). This allows us to find $\mu$,

$$
\mu=\frac{k_{B} T}{v_{0}} f_{m}^{\prime}\left(\phi_{0}\right)-\frac{1}{4} \Delta \rho \omega^{2}\left(R_{2}^{2}+R_{1}^{2}\right)
$$

where $f_{m}^{\prime}=d f_{m} / d \phi$. We notice that at the transition frequency and if $\phi<\phi_{c}=1 / 2$, the largest composition, found at $r=R_{2}$, crosses into the unstable part of the phase-diagram. This means that $\omega_{c}$ occurs when $\phi=\phi_{t}$ and $r=R_{2}$ are substituted in Eq. (5). The transition composition $\phi_{t}$ at a given Flory parameter $\chi$ (or temperature $T=B / \chi)$ is given by $N \chi=\ln \left(\phi_{t} /\left(1-\phi_{t}\right)\right) /\left(2 \phi_{t}-1\right)$, and hence we obtain the following expression for the critical demixing frequency

$$
w_{c}^{2}=\frac{4 k_{B} T}{v_{0} \Delta \rho} \frac{f_{m}^{\prime \prime}\left(\phi_{0}\right)}{R_{2}^{2}-R_{1}^{2}}\left(\phi_{t}-\phi_{0}\right)
$$

The notation $f_{m}^{\prime \prime}=d^{2} f_{m} / d \phi^{2}$ have been used. A similar expression exists for $\omega_{c}^{2}$ as a function of $T-T_{t}$.

A plot of $\omega_{c}$ as a function of the distance from the transition (binodal) temperature $T_{t}$ is shown in Fig. 3. $\omega_{c}$ increases as the temperature is elevated above $T_{t}$. If $\omega$ is larger than the plotted $\omega_{c}$, demixing occurs, otherwise the mixture displays only weak composition gradients. The numerical value of $\omega_{c}$ is quite low, and certainly achievable in conventional laboratory equipment $-\omega_{c}=60 \mathrm{rad} / \mathrm{sec}$ is equivalent to $572 \mathrm{rpm}$.

\section{Conclusion.}

Centrifugation of mixtures, whether of simple liquids or polymers, is shown here to be an effective means to induce a phase-separation transition. As is quite intuitive, composition variations occur at any rotation frequency, because of the force which appears due to the different densities of the mixture's components $[12,13,16,18]$. The simple mean-field free-energy model we use reveals a surprising phenomenon: for temperature lower than the critical temperature $T_{c}$, there exists a critical rotation frequency $\omega_{c}$. If the rotation is fast enough, $\omega>\omega_{c}$, a demixing transition occurs where the high density components is pulled toward the outer radius of the centrifuge, lower-density components are pulled in the other direction, and a sharp interface appears. At these high rotation frequencies, the centripetal force is large enough to cause large composition differences, which destabilize the homogeneous mixture in favor of a separated one. A somewhat similar phenomenon was predicted for liquid crystals under a gravitational field [8]. 


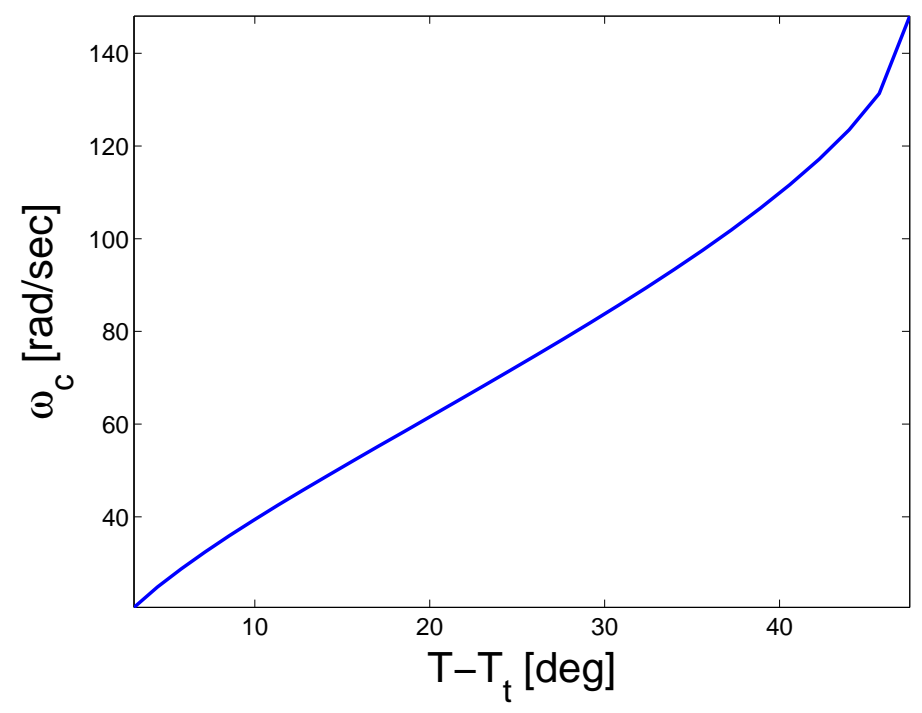

Figure 3. Critical rotation frequency $\omega_{c}$ for demixing, as obtained from Eq. (9). $T$ is the temperature and $T_{t}$ is the transition (binodal) temperature for demixing without centrifugation. We took $\phi_{0}=0.2$, and all other parameters are as in Fig. 1.

We note that demixing is also possible with spatially-varying electric fields. The wedge capacitor, made up of two flat electrodes tilted with respect to each other, is the analogue of the centrifuge. An electric field couples to the different permittivities of the liquid components $\Delta \varepsilon=\varepsilon_{A}-\varepsilon_{B}$. The electrostatic energy density is $\sim \Delta \varepsilon E^{2}$ and in this geometry the electric field varies like $r^{-1}[4,11]$. We thus find that $\Delta \rho$ plays the role of the permittivity difference $\Delta \varepsilon$, and $\omega r$ plays the role of an electric field. The critical voltage in the electrostatic case plays the same role as the critical frequency $\omega_{c}$ in the present case.

We obtained numerical solution for a closed rotating container with a fixed composition, as well as an approximate analytical expression which compares well with the numerical results. The results we find are not specific to the mixing free-energy model utilized, Eq. (3); the transition appears in other models which give a qualitatively correct phase-diagram in the absence of centrifugation. In addition, the $(\nabla \phi)^{2}$ term of Eq. (3) can be accounted for. It is expected to decrease the composition gradients and may be important close to the critical point. Numerical and analytical expressions for the case of an open system, having a connection to a liquid reservoir, and thus having an imposed chemical potential, are easier to obtain and are also at hand. However, to experimental setup is not straightforward [20], and we chose to leave it out.

The demixing behavior described above is quite different from the one commonly observed in laboratory experiments, where the slow sedimentation of a substance (e.g. polymers) in a solvent is simply accelerated by centrifugation. Our treaties show that even thermodynamically-stable mixtures of simple and complex fluids can be efficiently separated. It seems that this demixing transition can be used in many technological and chemical processes. For example, the reaction kinetics of two or more chemical species in a liquid environment can be controlled: if the mixture is homogeneous the reaction takes place everywhere, but when it is demixed the reaction can only take place at a thin boundary layer, and thus it has slower kinetics. Variations on this theme can also lead to accelerated kinetics: if one chemical species is an inhibitor for a reaction, after phase-separation it will have less contact with some of the liquid components, and the reaction can take place at a much higher rate.

Finally, we should stress that we used the simplest mean field model. This approximation can be relevant for systems far from critical point and indeed we have shown that even in such situations phase separation can be induced for rotation speeds easily attainable by modern centrifuges. The kinetics of phase separation and of formation of concentration profile within the sample seems to be an interesting open question that we leave until some experiments are performed.

Acknowledgements We are indebted to François Tournilhac for enthusiastic and stimulating discussions. We 
thank Professor Jean-Marie Lehn for sharing with us his thoughts of possible influence of gravitation forces on chemical equilibria and reactions. This research was supported by the Israel Science foundation (ISF) grant no. 284/05 and by the German-Israel Foundation (GIF) grant no. 2144-1636.10/2006.

\section{References}

[1] L.D. Landau, E.M. Lifshitz, Statistical Physics, Butterworth-Heinmann, New-York (2nd ed.), 1980.

[2] A. Onuki, H. Kitamura, J. Chem. Phys. 121 (2004) 3143-3151.

[3] A. Onuki, Phys. Rev. E 3 (2006) 021506-1-021506-16.

[4] Y. Tsori, F. Tournilhac, L. Leibler, Nature 430 (2004) 544-547.

[5] Y. Tsori, L. Leibler, Proc. Natl. Acad. Sci. (USA) 104 (2007) 7348-7350.

[6] Y. Tsori, D. Andelman, Interface Sci. 11 (2003) 259-268.

[7] Y. Tsori, Macromolecules 40 (2007) 1698-1702.

[8] V.A. Baulin, A.R. Khokhlov, Phys. Rev. E. 60 (1999) 2973-2977.

[9] J.X. Zhu, M. Li, R. Rogers, W. Meyer, R.H. Ottewill, W.B. Russell, P.M. Chaikin, Nature 387 (1997) 883-885.

[10] M. Sullivan, K. Zhao, C. Harrison, R.H. Austin, M. Megens, A. Hollingsworth, W.B. Russel, Z.D. Cheng, T. Mason, P.M. Chaikin, J. Phys. Cond. Mat. 15 (2003) S11-S18.

[11] Y. Tsori, L. Leibler, Phys. Rev. E. 71 (2005) 032101-1-032101-2.

[12] J.V. Sengers, J.M.J. Van Leeuwen, Physica A 116 (1982) 345-367.

[13] H.K. Leung, B.N. Miller, Phys. Rev. A 12 (1975) 2162-2167.

[14] S.C. Greer, M.R. Moldover, Ann. Rev. Phys. Chem. 32 (1981) 233-265.

[15] S. C. Greer, T.E. Block, C.M. Knobler, Phys. Rev. Lett. 34 (1975) 250-253.

[16] M.R. Moldover, J.V. Sengers, R.W. Gammon, R.J. Hocken, Rev. Mod. Phys. 51 (1979) 79-99.

[17] A. Boyum, Scandinavian Journal of Clinical \& Laboratory Investigation Suppl. 97 (1968) S21:77.

[18] S. Starobinets, V. Yakhot, L. Esterman, Phys. Rev. A 20 (1979) 2582-2589.

[19] S.A. Safran, Statistical Thermodynamics of Surfaces, Interfaces, and Membranes, Westview Press, Boulder, 2003.

[20] J. Mang, M. Ungarish, U. Schaflinger, Inter. J. Multi. Flow 27 (2001) 197-215. 